\title{
REPRÉSENTATIONS DE L'ALIMENTATION : CRISE DE LA CONFIANCE ET CRISES ALIMENTAIRES
}

Estelle Masson

Groupe d'études de psychologie | « Bulletin de psychologie »

2011/4 Numéro 514 | pages 307 à 314

ISSN 0007-4403

Article disponible en ligne à l'adresse :

https://www.cairn.info/revue-bulletin-de-psychologie-2011-4-page-307.htm

\section{Pour citer cet article :}

Estelle Masson, « Représentations de l'alimentation : crise de la confiance et crises alimentaires », Bulletin de psychologie 2011/4 (Numéro 514), p. 307-314. DOI 10.3917/bupsy.514.0307

Distribution électronique Cairn.info pour Groupe d'études de psychologie.

(c) Groupe d'études de psychologie. Tous droits réservés pour tous pays.

La reproduction ou représentation de cet article, notamment par photocopie, n'est autorisée que dans les limites des conditions générales d'utilisation du site ou, le cas échéant, des conditions générales de la licence souscrite par votre établissement. Toute autre reproduction ou représentation, en tout ou partie, sous quelque forme et de quelque manière que ce soit, est interdite sauf accord préalable et écrit de l'éditeur, en dehors des cas prévus par la législation en vigueur en France. Il est précisé que son stockage dans une base de données est également interdit. 


\section{Représentations de l'alimentation : MASSON Estelle* crise de la confiance et crises alimentaires}

\section{CONFIANCE ET REPRÉSENTATIONS DE L'ALIMENTATION}

La question de la confiance est une question particulièrement sensible, pour ne pas dire cruciale, dans les représentations de l'alimentation (Masson, Moscovici, 1997 ; Lahlou, 1998, Fischler, Masson, 2010). Manger, en effet, n'est pas un banal comportement de consommation, c'est bien plus. Manger c'est incorporer des nourritures, c'est les faire siennes. Et, comme le souligne Claude Fischler (1990), lorsqu'il décrit le processus d'incorporation, nous devenons ce que nous mangeons au sens littéral et au sens figuré. Nous devenons ce que nous mangeons sur le plan du réel, c'est-à-dire que nous transformons, par la digestion et l'assimilation, les aliments que nous consommons en notre propre matière. Cependant, nous devenons, aussi, ce que nous mangeons, au sens figuré, dans la mesure où, pour notre imaginaire, nous n'incorporons pas seulement les caractéristiques physiques, matérielles, biologiques des aliments, mais, également, tous les éléments symboliques associés à cet aliment. Ce deuxième aspect repose sur la pensée magique (Frazer, 1911).

Dans Les fonctions mentales dans les sociétés inférieures, Lévy-Bruhl (1910) rapporte de nombreux exemples, qui témoignent du fait que cette forme de pensée est à l'œuvre dans les croyances et les pratiques des primitifs. Ainsi, notamment, il rappelle qu'en Nouvelle-Zélande on faisait manger du Korimako (un oiseau particulièrement mélodieux) aux enfants, destinés à devenir chef, afin qu'ils soient éloquents et deviennent de bons orateurs, tandis que les habitants du Nord-Est de l'Inde pensaient que manger de la chouette permettait d'y voir clair la nuit... (Lévy-Bruhl, 1910 , p. 346). Mais la pensée magique n'est pas l'apanage des modes de pensée des membres des sociétés dites inférieures; elle est à l'œuvre aujourd'hui, aussi et encore, dans maintes de nos représentations relatives à l'alimentation, comme le montrent les résultats de la célèbre expérience de Nemeroff et Rozin (1989), menée auprès d'étudiants américains.
Le paradigme de l'expérience était simple: les auteurs proposaient, aux sujets participant à l'expérience, la description d'une population fictive, les Chandorans, prétendus habitants des îles Chandors, et leur demandaient d'évaluer les membres de cette population au moyen d'une série d'échelles bipolaires. La description contenait, entre autres, des informations sur les habitudes de chasse et les habitudes alimentaires de cette population. Selon les conditions expérimentales, seules, les habitudes alimentaires changeaient. Dans une condition expérimentale, il était dit que les Chandorans chassaient le sanglier pour en consommer la chair et chassaient la tortue uniquement pour sa carapace, c'està-dire qu'ils ne la mangeaient pas. Dans l'autre condition expérimentale, les Chandorans étaient présentés comme chassant, eux aussi, le sanglier et la tortue, mais la tortue pour la manger et le sanglier uniquement pour ses défenses. Selon les conditions expérimentales, les étudiants avaient donc à évaluer un peuple Chandoran soit mangeur de sanglier soit mangeur de tortue. Les résultats de l'expérience montrent que des membres de notre société occidentale ont, eux aussi, recours à la pensée magique lorsqu'il est question d'alimentation. Les mangeurs de tortue sont perçus, par les étudiants ayant participés à l'expérience, comme étant flegmatiques, bons nageurs, pacifiques..., tandis que les Chandorans mangeurs de sanglier sont perçus comme étant plus rapides à la course à pied, plus belliqueux, etc., en d'autres termes, les étudiants ont attribué les caractéristiques types des animaux mangés aux hommes qui les mangent.

Du fait de la spécificité du processus d'incorporation, manger n'est pas un banal comportement de consommation, car, les risques qu'encourt le mangeur lorsqu'il incorpore un aliment sont grands. En effet, manger un aliment, qui n'est pas bon, d'un point de vue bactériologique ou

* Centre de recherches en psychologie, cognition et communication (EA-1285), Université de Bretagne occidentale, CS 93837, 20 rue Duquesne, 29285 Brest Cedex 3.

<estelle.masson@univ-brest.fr> 
toxicologique, engendre des risques pour la santé (maladie, empoisonnement), mais, aussi, incorporer un aliment impur, un aliment, dont les propriétés symboliques, morales, sont incorrectes, engendre un risque de souillure (Douglas, 1967), c'est-à-dire constitue une menace pour l'identité du mangeur.

La confiance dans l'alimentation doit, donc, recouvrir simultanément deux niveaux: un premier, relatif à la qualité sanitaire des produits ; un second, relatif à la pureté des propriétés symboliques de l'aliment. Mais comment se construit cette double confiance et qu' advient-il de la « sécurité ontologique» (Giddens, 1987, p. 443) du mangeur, lorsque survient une crise alimentaire ?

\section{MÉFIANCE ET ALIMENTATION MODERNE}

Les contrôles sanitaires sur les aliments sont, aujourd'hui, beaucoup plus stricts qu'ils ne l'étaient par le passé et contribuent à faire, de l'alimentation moderne, une alimentation relativement sûre, d'un point de vue strictement sanitaire (Apfelbaum, 1998). Il apparaît, néanmoins, que le rapport de l'homme à l'alimentation moderne est marqué par un effritement, une dislocation, de la confiance (Poulain, 2002; Masson, Fischler, Laurens, Raude, 2003 ; Raude, 2008 ; Fischler, Masson, 2010). Des signes de la fragilité de cette confiance sont perceptibles dans différentes enquêtes récentes où nous avons abordé les représentations de l'alimentation.

Je m'appuierai, ici, sur les résultats d'enquêtes réalisées dans le cadre de deux recherches: une première recherche, portant sur la perception des risques relatifs à l'encéphalopathie spongiforme bovine (ESB) et à l'encéphalopathie subaiguë spongiforme transmissible (ESST) (Flahault, Masson, Fischler, Setbon, 2001) et une seconde recherche dans une perspective transculturelle du rapport à l'alimentation, au corps et à la santé (Fishler, Masson, 2008). Dans la première recherche, une enquête quantitative a été réalisée en janvier 2001 (c'est-à-dire au lendemain de la deuxième crise de la vache folle), auprès d'un échantillon représentatif de la population française $(\mathrm{n}=916)$. La seconde recherche, menée de 2000 à 2002, comprenait une phase qualitative et une phase quantitative. Durant la phase qualitative, des groupes centrés ont été réalisés dans sept pays différents: Allemagne, Suisse, Royaume-Uni, États-Unis d'Amérique, France, Italie et Inde. La phase quantitative comportait deux vagues: une première enquête, réalisée en septembre-octobre 2001, qui a porté sur un un échantillon $(\mathrm{n}=1032)$ restreint et raisonné ( $1 / 3$ consommateurs, $1 / 3$ enseignants et $1 / 3$ médecins), sélectionné dans les six pays occidentaux participant à la recherche. Cette première enquête, par questionnaire (administré par téléphone), conçue dans une perspective comparative intra- et inter-culturelle, comportait de très nombreuses questions ouvertes et visait à tester l'existence d'homogénéités transculturelles et de spécificités locales (par exemple: en matière d'alimentation, les vérités médicales sont-elles culturellement dépendantes ou le savoir médical transcende-t-il les frontières ?) Les résultats obtenus ont été affinés et validés lors d'une deuxième enquête, réalisée en février-mars 2002, auprès de 6023 personnes, réparties en 6 souséchantillons nationaux représentatifs (effectifs: environ 900 individus dans chacun des pays européens et 1500 aux États-Unis).

L'analyse des résultats obtenus lors de ces différentes recherches révèle de nombreux signes de la fragilité de la confiance dans le rapport de l'homme à l'alimentation moderne. Ainsi, par exemple, dans la première enquête de la recherche transculturelle, où il avait été demandé aux répondants d'évaluer en gains ou en pertes, par rapport au passé, différents aspects relatifs à l'alimentation, on observe (voir tableau 1) que $57 \%$ des personnes interrogées jugent qu'en matière d'alimentation, on a perdu en confiance, par rapport au passé. Et c'est en France que ce sentiment de perte est le plus largement répandu : $64 \%$ des Français déclarent le penser. Ce résultat ne saurait être interprété comme le simple indice d'un rapport nostalgique à l'alimentation (Masson 2002), car, comme le montrent les autres résultats, on décèle l'existence d'une perception contrastée de l'évolution de l'alimentation.

En effet, une large majorité des répondants s'accorde pour reconnaître, notamment, une évolution positive en matière d'hygiène, concède qu'il y a aujourd'hui beaucoup plus de choix que par le passé, et même la majorité des Britanniques considère que la qualité de l'alimentation s'est améliorée au fil des ans. Nonobstant, les mangeurs ne font plus - ou ont le sentiment de ne plus pouvoir faire confiance à leur alimentation.

C'est comme si, avec, en particulier, l'industrialisation des processus de production et de transformation des aliments, la proportion entre savoir et non savoir devenait trop importante (Simmel, 1908/1999, p. 356-357), autant d'éléments que l'on retrouve implicitement dans le sentiment de perte de la tradition partagé par plus des deux tiers des Français et de l'ensemble des personnes interrogées dans cette enquête. Les réticences face aux organismes génétiquement modifiés (OGM) (Fischler, Raude, 2005) sont symptomatiques de ce phénomène, car un aliment génétiquement modifié (c'est-à-dire un aliment, dont l'identité a été modifiée par le biais d'une manipulation génétique) conserve l'apparence de ce qu'il prétend être, mais, 


\begin{tabular}{|c|c|c|c|c|c|c|c|}
\hline & & Hygiène & Choix & Goût & Qualité & Tradition & Confiance \\
\hline \multirow[t]{2}{*}{ France } & gagné & 81 & 90 & 14 & 36 & 7 & 25 \\
\hline & perdu & 16 & 8 & 67 & 51 & 68 & 64 \\
\hline \multirow[t]{2}{*}{ Italie } & gagné & 82 & 84 & 27 & 36 & 8 & 38 \\
\hline & perdu & 13 & 9 & 54 & 51 & 74 & 51 \\
\hline \multirow[t]{2}{*}{ Suisse } & gagné & 80 & 89 & 27 & 40 & 9 & 17 \\
\hline & perdu & 12 & 8 & 59 & 40 & 74 & 66 \\
\hline \multirow[t]{2}{*}{ Allemagne } & gagné & 74 & 84 & 40 & 45 & 16 & 18 \\
\hline & perdu & 9 & 8 & 32 & 30 & 56 & 54 \\
\hline \multirow[t]{2}{*}{ Royaume-Uni } & gagné & 74 & 94 & 39 & 50 & 12 & 12 \\
\hline & perdu & 12 & 1 & 39 & 25 & 72 & 61 \\
\hline \multirow[t]{2}{*}{ États-Unis } & gagné & 65 & 83 & 14 & 47 & 13 & 26 \\
\hline & perdu & 16 & 8 & 25 & 31 & 66 & 44 \\
\hline \multirow[t]{3}{*}{ Total } & gagné & 76 & 87 & 31 & 42 & 11 & 23 \\
\hline & perdu & 13 & 7 & 46 & 38 & 68 & 57 \\
\hline & Rien n'a changé & 11 & 6 & 23 & 20 & 21 & 21 \\
\hline
\end{tabular}

Tableau 1. Gains et pertes par rapport au passé ( $\%$ des réponses).

pour partie, tout au moins, par essence, n'est plus celui qu'il prétend être. Quel est-il alors ? Les recherches menées sur les représentations des OGM montrent que, bien souvent, un tel aliment est perçu comme «inclassable» et relevant du monstrueux (Wagner, Kronberger, 2002). Quels risques identitaires encourt le mangeur en l'incorporant ? En quoi risque-t-il de se «transformer»? Autant de questions qui, sur un plan fantasmagorique, restent en suspens et contribuent au rejet de ces aliments. Dans la deuxième enquête quantitative de la recherche transculturelle, $87 \%$ des Français se déclarent opposés à «l'utilisation d'organismes génétiquement modifiés dans l'alimentation ».

La fragilité de la confiance du mangeur contemporain a, également, été mesurée et constatée dans l'enquête sur la perception des risques. $82 \%$ des 916 répondants s'accordent à dire que «de nos jours on ne sait plus ce que l'on mange » et $67 \%$ d'entre eux considèrent qu' " aujourd'hui on ne sait plus quoi acheter pour ne pas s'empoisonner».

On constate donc bien une sorte de méfiance de l'homme moderne à l'égard de son alimentation. Une méfiance diffuse, qui, en temps normal, n'est cristallisée sur aucun aliment en particulier, mais concoure à faire apparaître, indistinctement, tous les aliments suspects. Dans un tel contexte, que se passe-t-il lorsqu'un nouveau risque alimentaire est découvert? Que se passe-t-il lorsque des découvertes scientifiques jettent le doute sur un aliment spécifique? Que se passe-t-il lorsque, de surcroît, l'aliment incriminé est un élément relativement central dans le régime alimentaire d'une population?

La réponse est simple, nous la connaissons : une crise survient et, qui plus est, une crise importante, si on la mesure à l'aune des indicateurs économiques comme, par exemple, la chute des consommations, la chute du cours du produit, etc. Ce phénomène a pu être observé, lors de l'annonce de la transmissibilité possible à l'homme de la maladie de la vache folle, en 1996 : c'est la première crise, dite de la vache folle, mais c'est, aussi, ce qui s'est passé en 2000, lorsqu'une vache malade a été détectée à l'entrée d'un abattoir et que la maladie s'est dangereusement approchée de l'assiette des mangeurs.

\section{Vache folle: risques identitaires et risques sanitaires}

L'entrée de la vache folle dans le champ social ne s'est pas limitée à une crise de confiance, relative à la qualité sanitaire de la viande. Elle s'est doublée de diverses prises de conscience, venant, elles aussi, ébranler un peu plus encore ce rapport, déjà fragile, de confiance du mangeur contemporain à ce qu'il mange. La crise de la vache folle est 
une crise de confiance où, comme nous allons le voir, les craintes identitaires et les craintes pour la santé surgissent et s'entremêlent, en suite de la modification des savoirs et des connaissances dont disposent les mangeurs sur le bœuf.

Les craintes sanitaires sont directement liées à la découverte d'un nouveau danger: la possibilité d'être contaminé par la consommation de certains produits d'origine bovine est affirmée par des scientifiques et reconnue par les autorités publiques. Les résultats de l'enquête de 2001 montrent que la peur de la contamination existait véritablement : $65 \%$ des personnes interrogées se déclaraient préoccupées pour elles-mêmes ou pour leurs enfants par la maladie de la vache folle. La crainte d'avoir été contaminé dans le passé était, elle aussi, à ce moment, assez largement répandue $(45 \%$ se disaient d'accord avec la proposition « j'ai de quoi m'inquiéter car je suis peut-être déjà contaminé par la maladie »).

Cependant, la peur de la maladie n'explique pas tout. En effet, on observe, d'une part, que l'innocuité des tissus musculaires, c'est-à-dire de la viande, affirmée par les scientifiques, était acceptée par une large majorité de mangeurs : près des deux tiers ne pensaient pas que la viande de bœuf (le muscle) était susceptible de transmettre la maladie ; d'autre part, $68 \%$ des répondants déclaraient que les morceaux potentiellement dangereux n'étaient plus vendus, et $61 \%$ des personnes interrogées affirmaient que le risque, pour l'homme, d'être contaminé, était très faible. Autant d'éléments convergents, témoignant d'une crainte concrète d'être contaminé directement, lors de la consommation de viande d'origine bovine, somme toute réduite, qui rendent difficilement explicable, par le seul argument de la peur de la contamination, le fait que $84 \%$ des personnes interrogées reconnaissaient, en 2001, avoir opéré des modifications dans leur régime alimentaire, depuis la survenue de la maladie de la vache folle.

Pour comprendre l'ampleur de la crise, il apparaît donc nécessaire de ne pas se limiter à une explication par la peur de la contamination et de la maladie, mais, aussi, de prendre en compte le fait que la crise de la vache folle comportait, également, de nombreuses dimensions éthiques, politiques et symboliques.

En effet, l'annonce, en mars 1996, de la transmissibilité de l'encéphalopathie spongiforme bovine (ESB) à l'homme ne se réduit pas à la reconnaissance officielle d'un nouveau danger; pour le public, elle correspond, également, à l'émergence dans le champ social d'un ensemble de faits et de phénomènes qui, jusqu'alors, étaient restés cachés ou, plutôt, étaient restés invisibles pour le grand nombre. Et les différentes révélations, qui ont été faites au public, ont ébranlé le champ des savoirs, des croyances et des connaissances relatifs à la viande.

Tout d'abord, un certain nombre de personnes ont appris, avec stupéfaction, qu'elles mangeaient de la vache et non du bœuf. Ensuite, en prenant connaissance des causes de l'apparition de la maladie, à savoir l'utilisation de farines animales contaminées dans l'alimentation du bétail, les consommateurs découvrirent, avec plus de stupéfaction encore, que le régime alimentaire d'animaux, qu'ils considéraient être exclusivement herbivores, comprenait, également, depuis bien des décennies déjà, des éléments d'origine animale. Comprise comme l'aveu qu'une transgression suprême de l'ordre naturel avait été commise, cette révélation souleva l'indignation : $87 \%$ des répondants jugeaient qu'il était choquant d'avoir « rendu des herbivores carnivores ». Les éclaircissements qui, progressivement, ont été apportés sur les dites farines animales laissèrent, elles, apparaître une composition des plus douteuses pour un public néophyte. Ayant ingéré ces farines composées, en partie, des restes recyclés de leurs congénères, les vaches se révélaient, donc, non seulement carnivores, mais, de surcroît, cannibales. Et, en vertu du processus d'incorporation et de la règle de transitivité qui le sous-tend, on peut se demander, avec Kilani (2002), si « le cannibalisme de la vache que l'homme met dans son assiette, n'induit pas chez lui le même comportement cannibale » ou, tout au moins, génère chez lui l'horreur de cette idée, rendue plus présente encore par les rapprochements qui furent établis par les scientifiques (Schwartz, 2001) entre « la maladie de la vache folle » et le Kuru, cette maladie mortelle, ayant sévi en Nouvelle-Guinée et dont la diffusion était liée précisément à des pratiques cannibales.

La crise a donc révélé, au public, des processus industriels, qui ont été interprétés comme étant une transgression de l'ordre naturel (des herbivores rendus carnivores), mais, aussi, comme étant contraires à l'éthique et à la morale (irrespect de la vie animale, égoïsme, mise en danger d'autrui, etc.). Dans l'enquête réalisée, en 2001, $81 \%$ des personnes interrogées considéraient que la maladie de la vache folle était liée à « une recherche aveugle de profit »; $91 \%$ jugeaient que l'on se souciait «plus du profit que des risques pour la population ».

Autant de révélations qui ont contribué, pour de nombreux mangeurs, à une perception de la viande d'origine bovine comme étant un aliment impur, un aliment porteur et vecteur de souillure. Autant de révélations qui ont pu être interprétées comme des preuves de la trahison de la confiance. Et on peut se demander dans quelle mesure ne plus manger de viande, c'est-à-dire bouder la viande n'a 
pas été, aussi, un moyen, pour les consommateurs, de signifier à autrui qu'ils savaient qu'il avait trahi leur confiance?

\section{La confiance trahie}

Mais qui est cet autrui, qui est cet autre, ce « on » accusé d'avoir abusé de la confiance des mangeurs ? Il apparaît que ce sont, non seulement, tous les acteurs ayant directement «participé » à la transgression de l'ordre naturel (les industriels, les éleveurs, etc.), ainsi que les institutions, les autorités publiques, qui, jusqu'alors, avaient été implicitement pensées comme les garantes de la qualité sanitaire des produits mis en vente sur le marché.

Les résultats de l'enquête portant sur la perception $\mathrm{du}$ risque d'ESB et d'encéphalopathie subaiguë spongiforme transmissible (ESST) montrent, là encore, l'idée, partagée par une large majorité des répondants, que la confiance, qui, naguère, avait été octroyée à l'autorité publique se révélait injustifiée, du fait que cette dernière était, au lendemain de la crise, perçue comme n'ayant su gérer le problème ni convenablement, ni au bon moment. En effet, $83 \%$ des personnes questionnées se déclaraient d'accord avec la proposition : «le gouvernement a trop tardé à prendre les mesures nécessaires » et, selon $71 \%$, il «n'a pas su prendre les bonnes mesures au bon moment ». L'action de la communauté européenne était, elle aussi, remise en question : $76 \%$ jugeaient, en effet, qu'elle n'avait, elle non plus, su prendre les bonnes mesures au bon moment. De plus, le sentiment que l'autorité publique était soumise aux pressions de groupes, ayant des intérêts divergents de ceux des mangeurs, était largement répandu. Certains groupes tenteraient de corrompre, de pervertir l'autorité politique et y parviendraient, comme le suggèrent les $76 \%$ des personnes interrogées, qui considéraient que «les lobbies font pression pour empêcher certaines mesures d'être prises ».

Dans un tel contexte, quels étaient les acteurs sociaux, à qui les mangeurs pensaient encore pouvoir accorder légitimement leur confiance, quels étaient les acteurs sociaux jugés dignes de foi ? Dans l'enquête sur la perception des risques, il a été demandé aux répondants d'évaluer, au moyen d'une note comprise entre 0 et 10 , la confiance qu'ils accordaient aux informations diffusées par différentes sources, par différents « autrui » signifiants dans ce contexte. L'analyse des résultats (voir tableau 2) montre que les personnes interrogées déclaraient, en moyenne, accorder peu de crédit, d'une part, aux informations émanant des services des différents ministères, directement intéressés par la crise (et il est notable que les informations émises, par le ministère de l'Agriculture, suscitaient beaucoup de défiance); d'autre part, aux informations diffusées par les médias, c'est-à-dire celles auxquelles les consommateurs avaient principalement accès. Mais, ce qui est frappant, c'est que ce sont les informations diffusées par les hommes politiques et les élus, c'est-à-dire par les représentants élus démocratiquement des citoyens, qui étaient perçues comme étant les moins fiables, voire les plus suspectes.

\begin{tabular}{lc}
\hline Source & $\begin{array}{c}\text { Note } \\
\text { (moyenne sur 10) }\end{array}$ \\
\hline Les associations de consommateurs & 7,1 \\
\hline Votre médecin & 6,9 \\
\hline Les experts scientifiques & 6,9 \\
\hline L'agence française pour la sécurité & 6,3 \\
alimentaire ou AFSSA & 6,1 \\
\hline Les bouchers & 5,5 \\
\hline Les éleveurs & 5,5 \\
\hline Les services du ministère de la Santé & 5,4 \\
\hline Les services du ministère de la & \\
\hline Consommation & 4,9 \\
\hline \begin{tabular}{l} 
l'Agriculture \\
\hline Les journalistes, la presse, les médias
\end{tabular} & 4,5 \\
\hline Les hommes politiques et les élus & 2,9 \\
\hline
\end{tabular}

Tableau 2. Classement des sources d'information selon la confiance qui leur est accordée.

Le niveau de confiance accordé aux informations émises par une source semble déterminé par les facteurs suivants: l'indépendance perçue de la source vis-à-vis de la gestion de l'épidémie, la reconnaissance de son expertise scientifique et technique, mais, aussi et surtout, sa proximité (subjective ou physique) avec les consommateurs.

Ainsi, les instances et acteurs jugés les plus dignes de foi sont les associations de consommateurs, son propre médecin et les experts scientifiques. Il est frappant de constater que les répondants attribuaient, en moyenne, une note de fiabilité à peine inférieure aux informations émises par leurs bouchers qu'à celles diffusées par l'Agence française de sécurité sanitaire des aliments (AFFSA); nous reviendrons, plus loin, sur la question de la confiance octroyée au boucher.

\section{Comment se protéger ou que faire dans un contexte ou la confiance fait défaut ?}

Dans l'enquête sur la perception des risques, on s'était également interrogé sur la manière dont les 
individus opéraient pour gérer personnellement le nouveau risque. Le premier constat, qui s'impose, est que la majorité des personnes interrogées pensait que les risques, liés à la maladie de la vache folle, ne pouvaient pas être maîtrisés par l'action individuelle $(57 \%$ des personnes interrogées ne pensaient pas que la maladie puisse être évitée par des précautions personnelles) et, pourtant, l'analyse des données de l'enquête montre que la majorité des répondants avait élaboré des stratégies visant à réduire les risques de contamination. La gestion individuelle du risque passait, d'une part, par les évitements alimentaires et, d'autre part, par le contrôle des approvisionnements.

En 2001, les évitements alimentaires apparaissaient être le pivot de la gestion individuelle du risque de contamination. L'indice synthétique de changement montre que $84 \%$ des personnes interrogées reconnaissaient avoir modifié leurs consommations alimentaires depuis l'apparition de la maladie de la vache folle, et $66 \%$ déclaraient qu'il était légitime de réduire sa consommation de bœuf, du fait qu'on ne disposait pas de toutes les connaissances nécessaires pour se prémunir du risque.

Le choix de la viande, au moment de l'achat, était envisagé, par la majorité des personnes interrogées, comme un moyen susceptible de réduire le risque d'être contaminé. Les viandes «certifiées » étaient perçues comme moins dangereuses. Ainsi, $62 \%$ des répondants jugeaient que l'on prenait moins de risques en achetant de la viande « label rouge », $58 \%$ que l'on prenait moins de risques en achetant de la «viande bovine française », $56 \%$ que l'on prenait moins de risques en achetant de la viande « bio ».

Mais, c'est la sélection du fournisseur, qui apparaît être le moyen perçu comme étant le plus efficace pour réduire son exposition personnelle au risque. Et on retrouve, là, la personne du boucher, dont la bonne position dans le classement des sources d'informations a déjà été mentionnée plus haut : $65 \%$ des répondants pensaient, en effet, que l'on prenait moins de risques en achetant de la viande bœuf «chez un boucher que l'on connaît ».

Mais, pourquoi acheter sa viande en boucherie, chez un boucher que l'on connaît, protègerait contre la maladie de la vache folle ? Pourquoi la viande, vendue par cet homme, serait-elle moins dangereuse, alors qu'il ne dispose d'aucun outil technique spécifique (comme, par exemple, de tests décelant la présence ou l'absence de l'agent contaminant), pour s'assurer des propriétés sanitaires de sa marchandise?

Il y a, certainement, l'idée que la viande qu'il vend est de meilleure qualité, car, plus chère et, aussi, que ce surcoût, par rapport au prix de la viande vendue en supermarché, garantissait l'absence, sur son étal, de viande provenant de vaches de réformes, c'est-à-dire de la catégorie des animaux présentée comme étant susceptible d'être la plus dangereuse. Cet aspect soulevait, d'ailleurs, comme nous avons pu le constater, lors de groupes centrés, la question d'une inégalité sociale dans l'exposition au risque.

Ce qui se profile surtout derrière les résultats concernant le boucher c'est le besoin, en période de crise, d'un retour à la connaissance personnelle. La réputation du boucher apparaît plus efficace pour restaurer la confiance que les mesures sanitaires prises par le gouvernement. Il semble que les mangeurs aient été disposés à faire confiance à leur boucher, parce que, précisément, il s'agissait de leur boucher et qu'ils le connaissaient. Et c'est comme si la connaissance qu'ils avaient de leur boucher, d'un point de vue personnel (un homme souvent présenté comme jovial - dont l'épouse, Madame la bouchère, est elle-même parfois connue du mangeur-, qui indique les bons temps de cuisson, qui a pignon sur rue depuis plusieurs années, et qui, à la connaissance du sujet, n'a jamais empoisonné personne ; il n'a rien d'un monstre...) devenait le support de la confiance octroyée à la viande ayant été en contact avec lui. Cette connaissance personnelle du boucher est assortie de la supposition que toute la chaîne menant de l'animal au mangeur est jalonnée par une connaissance du même ordre : le boucher est présumé connaître ses fournisseurs qui, idéalement, dans la représentation des mangeurs, sont des éleveurs, et si tel n'est pas le cas et qu'un intermédiaire est envisagé, que cet intermédiaire, connu du boucher, connaît, lui, personnellement l'éleveur, qui, lui-même, connaît l'animal, dont provient la viande et sait de quoi il a été nourri. C'est cette connaissance personnelle d'individu à individu, balisant le trajet de l'animal au mangeur, qui apparaît comme le support de la confiance; c'est elle qui tient lieu de garantie qu'aucun des acteurs n'ait été monstrueux, c'està-dire n'ait participé à la transgression, jugée abominable, d'avoir rendu carnivores des herbivores. On retrouve ici l'idée, développée par Giddens, selon laquelle, «dans le cas de la confiance envers des agents humains, la présomption de fiabilité implique l'attribution de "probité" (honneur) ou d'amour» (Giddens, 1994, p. 40). La confiance accordée au boucher (qui, comme par ricochet, s'étend aux fournisseurs, auxquels le boucher est supposé lui-même faire confiance) fonctionne, pour le mangeur, comme la garantie du respect des règles morales et de l'ordre naturel, qui, dans le cas de la maladie de la vache folle, devenait, aussi, la garantie supposée d'une viande non contaminée. 


\section{CONCLUSION : CRISE DE CONFIANCE ET CRISE DU LIEN SOCIAL}

La crise de la vache folle montre que, lorsque les mécanismes habituels de la confiance, au sein d'une société, sont ébranlés, la forme de confiance qui résiste le mieux est celle qui relie directement un individu à un autre et comporte une dimension charnelle ; elle oppose, ainsi, la confiance publique à la confiance interindividuelle. La crise de la confiance à l'égard de la viande, révélait de ce fait, aussi, une crise du lien social, où les citoyens manifestaient leur défiance, d'une part, à l'égard de l'autorité publique et de leurs représentants élus démocratiquement; d'autre part, à l'égard des médias. Ni les mesures prises par le gouvernement, ni même les démonstrations publiques de consommation de l'aliment suspect, faites par des hommes publics devant les caméras de télévisions, n'étaient susceptibles de restaurer la confiance des consommateurs, sans une restauration et un resserrement du lien social.

Le temps que dura la crise de la vache folle, c'est comme si, pour faire confiance, il était devenu nécessaire de repasser par l'action réciproque et la connaissance personnelle. C'est comme si, pour un temps, il avait été nécessaire de revenir à une forme plus archaïque de la confiance, celle marquant ces " sociétés plus primitives et moins différenciées, [où selon Simmel] on en savait beaucoup plus sur son partenaire du point de vue personnel - et beaucoup moins quant à la possibilité purement objective de lui faire confiance » (Simmel, 1908/1999, p. 356-357). Il est fort probable que si, lors de la crise, la viande, vendue par un boucher connu du mangeur, était jugée moins suspecte que celle achetée en libre-service, dans les supermarchés, c'est, précisément, parce qu'elle était vendue par un individu jugé, par un autre individu, digne de foi et qui apportait une caution personnelle (et non sanitaire) au produit. La viande issue des circuits de la grande distribution ne bénéficiait, elle, d'aucune caution de ce type. La confiance accordée au boucher «appartient à ces états psychiques et sociaux qui forgent des liens tenant à la fois du devoir et du sentiment » (Watier, 2002, p. 236) et relève de « ce domaine des liens sociaux régis par des obligations réciproques » (p. 235). Dans ce domaine prévaut une forme de rationalité particulière: la «rationalité fiduciaire», comme la nomme Serge Moscovici lorsqu'il développe l'idée de « une culture, deux rationalités » (Moscovici, 1993).

Transposé dans la perspective théorique d'Antony Giddens, le boucher apparaît comme un point d'accès au système abstrait, qu'est, pour tout consommateur lambda, la filière alimentaire (Lambert, 1987). "Ces points d'accès au système abstraits constituent un terrain pour des engagements en face à face » (Giddens, 1994, p. 89) et la personnalisation de ces points d'accès, conformément à l'intuition de Giddens, semble jouer un rôle important pour la restauration de la confiance accordée au système.

Dans les faits, ce besoin d'un retour à une confiance, fondée sur la connaissance personnelle, s'est traduit par le développement de l'intérêt, témoigné par les consommateurs, pour la notion de traçabilité. Cependant, la législation mise en place, par les pouvoirs publics, sur la question n'était pas à même de satisfaire pleinement les consommateurs. Elle permettait, certes, de rétablir un lien entre le produit vendu et l'animal, mais se matérialisant par des numéros d'abattoirs, de lots, etc., cette possibilité objective était réservée à une minorité d'experts. Ce qu'auraient souhaité les consommateurs, c'est une traçabilité de la catégorie des animaux («de réforme» ou «race à viande»), mais, aussi et surtout, des individus, afin de savoir à qui ils avaient à faire confiance. La crise, dite de la vache folle, est aujourd'hui passée ; la consommation de viande de bœuf a repris et après l'effondrement, qui dura le temps de la crise, le montant des dépenses (en euros constants), en France, en viande de bœuf dépassent, depuis 2003, les montants antérieurs à la crise. Cependant, le besoin de faire confiance demeure et, se détachant de la viande de bœuf, en particulier, et s'étendant simultanément à d'autres denrées, il revêt de nouvelles formes. Le développement des associations pour le maintien d'une agriculture paysanne (AMAP), l'apparition et le développement de la nouvelle tendance «locavore », qui consiste à rechercher et à privilégier des nourritures cultivées et transformées dans une aire géographique restreinte et proche du consommateur, vont dans ce sens. En effet, la proximité et la réduction du nombre des intermédiaires entre producteurs et consommateurs, contribuent, pour le consommateur, à la possibilité effective d'une connaissance personnelle du producteur. 


\section{RÉFÉRENCES}

ApFelbaum (Marian).- Risques et peurs alimentaires, Paris, Odile Jacob, 1998.

Douglas (Mary).- De la souillure. Essai sur les notions de pollution et de tabou [1967], trad. fr., Paris, Éditions de la découverte, 1992.

Flahault (Antoine), Masson (Estelle), Fischler (Claude), Setbon (Michel).- Perception du risque d'ESB et d'ESST : déterminants et variabilité, Rapport d'activité au 15 novembre 2001, INSERM/CNRS, 2001.

FISCHLER (Claude).-L'homnivore, Paris, Odile Jacob, 1990.

FISCHLER (Claude), MASSON (Estelle).-Manger. Français, Européens et Américains face à l'alimentation, Paris, Odile Jacob, 2008.

Fischler (Claude), MASson (Estelle).- Désenchantement et réenchantement de l'alimentation, dans Apfeldorfer (G.), Waysfeld (B.) Zermati (J.-P.), Traiter l'obésité et le surpoids, Paris, Odile Jacob, 2010, p. 346-377.

FISCHLER (Claude), RAUDE (Jocelyn).- La percepcio dels aliments genèticament modificats a occident : divergència i/o convergència, Revista d'etnologia de Catalunya, 27, 2005, p. 100-111.

Frazer (James George).- Le Rameau d'Or, Vol. 1 [1911], trad. fr., Paris, Robert Laffont, 1981.

GIDDENS (Anthony).- La constitution de la société, Paris, Presses universitaires de France, 1987.

GidDENS (Anthony).- Les conséquences de la modernité, Paris, L'harmattan, 1994.

KILANI (Mondher).- Crise de la «vache folle » et déclin de la raison sacrificielle, Terrain, 38, 2002, p. 113-126.

LAHLOU (Saadi).- Penser manger, Paris, Presses universitaires de France, 1998.

LAMBERT (Jean-Louis).- L'évolution des modèles de consommation alimentaire en France, Paris, Lavoisier, 1987.

LÉVY-BRUHL (Lucien).- Les fonctions mentales dans les sociétés inférieures [1910], Paris, Alcan, 1951.
MASson (Estelle).- Les nourritures de la mémoire et de l'identité, dans Laurens (S.), Roussiau (N.), La mémoire sociale, identités et représentations sociales, Rennes, Presses universitaires de Rennes, 2002, p. 279-286.

Masson (Estelle), Fischler (Claude), LAurens (Stéphane), RAUdE (Jocelyn).- La crise de la vache folle : «psychose», contestation, mémoire et amnésie, Connexion, 80, 2, 2003, p. 89-100.

Masson (Estelle), Moscovici (Serge).-Les mutations dans la pratique alimentaire. Processus symboliques et représentations sociales, Rapport de fin de recherches, Paris, École des Hautes études en sciences sociales / Ministère de l'Agriculture, 1997.

Moscovici (Serge).- Razón y culturas, dans Moscovici (S.), Barriga (S.), Arte la Nueva Europa, Seville, Mimeo, 1993, p. 30-62.

Nemeroff (Carol), Rozin (Paul).- « You are what you eat » : applying the demand-free «impressions» technique to an unacknowledged belief, Ethos, 17, 1, 1989, p. $50-69$.

Poulain (Jean-Pierre).- Sociologies de l'alimentation, Paris, Presses universitaires de France, 2002.

RAUDE (Jocelyn).- Sociologie d'une crise alimentaire. Les consommateurs à l'épreuve de la maladie de la vache folle, Paris, Lavoisier, 2008.

SCHWARTZ (Maxime).-Comment les vaches sont devenues folles, Paris, Odile Jacob, 2001.

SIMMEL (Georg).- Sociologie. Études sur les formes de la socialisation [1908], trad. fr., Paris, Presses universitaires de France, 1999.

WAGNer (Wolfgang), Kronberger (Nicole).Mémoires des mythes vécus. Représentations de la technologie génétique, dans Laurens (S.), Roussiau (N.), La mémoire sociale, identités et représentations sociales, Rennes, Presses universitaires de Rennes, 2002, p. 83-93.

WATIER (Patrick).- La place des sentiments psychosociaux dans la sociologie de G. Simmel, dans DerocheGurcel (L.), Watier (P.), La sociologie de Georg Simmel (1908). Éléments actuels de modélisation sociale, Paris, Presses universitaires de France, 2002, p. 217-239. 